\title{
Nuclear Magnetic Resonance Microscopy for In Vivo Metabolomics, Digitally Twinned by Computational Systems Biology, Needs a Sensitivity Boost
}

\author{
Jan Gerrit Korvink, "Vlad Badilita, Lorenzo Bordonali, \\ Mazin Jouda, Dario Mager, and Neil MacKinnon \\ Institute of Microstructure Technology, Karlsruhe Institute of Technology, \\ Hermann-von-Helmholtz-Platz 1, 76344 Eggenstein-Leopoldshafen, Germany
}

(Received July 26, 2017; accepted September 26, 2017)

Keywords: nuclear magnetic resonance, magnetic resonance imaging, Caenorhabditis elegans, microcoils, microresonators

The metabolism of an organism is regulated at the cellular level, yet is strongly influenced by its environment. The precise metabolomic study of living organisms is currently hampered by measurement sensitivity; most metabolomic measurement techniques involve some compromise, whereby averaging is performed over a volume significantly larger than a single cell, or require the invasion of the organism, or an arrested state of the organism. Nuclear magnetic resonance (NMR) is an inherently noninvasive chemometric and imaging method, and hence, in principle, suitable for metabolomic measurements. The digital twin of metabolomics is computational systems biology, such that NMR microscopy is potentially a viable approach to joining the theoretical and experimental explorations of the metabolomic and behavioural responses of organisms. In this study, we consider the challenge of performing in vivo NMRbased metabolomics on the small organism Caenorhabditis elegans, point the way towards possible solutions created using techniques specific to micro-electromechanical systems (MEMS) fabrication, and highlight currently insurmountable challenges.

\section{Introduction}

Metabolomics is the study of the molecules of life, as expressed by the reagents and products of biochemical reactions taking place within the cells of a living organism. The open-access Kyoto Encyclopedia of Genes and Genomes (KEGG) pathway database ${ }^{(1,2)}$ describes the known metabolomic pathway maps for a range of organisms, linking and organising contributions from the scientific literature. An organism metabolome is a time-dependent fingerprint of the state of a cell, and full knowledge of the metabolome would, in principle, reveal many cellular-level upstream mechanisms that form part of the omic chain (genomics, transcriptomics, proteomics, and so on), and link them with downstream behavioural responses. It is a key premise of

*Corresponding author: e-mail: jan.korvink@kit.edu http://dx.doi.org/10.18494/SAM.2018.1711 
systems biology ${ }^{(3)}$ that the metabolome can be computed via a partial differential equation system for the joint metabolomic pathways, and practitioners aim to use the results to predict organism responses to disease or environmental influences. To date, no general noninvasive method of measuring the instantaneous metabolome of sufficient resolution in space, time, species, or rate exists. In fact, the metabolomic literature is rife with measurements by mass spectrometry, which completely destroys the sample by vapourisation, and may require an extensive pretreatment such as chromatography. Nuclear magnetic resonance (NMR) is a noninvasive alternative to mass spectroscopy for extracting detailed information about the metabolic composition of a target sample. On the one hand, the powerful analytical capabilities of NMR are derived from the high availability of specialised pulse sequences and methods that allow for the extremely granular investigation of a metabolic profile. On the other hand, the technique suffers from an inherently limited sensitivity, which renders the feasibility of even simple experiments progressively more challenging as the sample volume (or metabolite concentration) is reduced towards the $\mu \mathrm{L}$ or $\mathrm{nL}$ range ( $\mu \mathrm{M}$ or $\mathrm{nM}$ concentrations). Hence, a methodology of verifying the predictions of systems biology, the digital twin of experimental metabolomics, and behavioural studies is currently lacking. In this paper, the need for this experimental capability is detailed, and some solutions that hold promise for enabling in vivo NMR metabolomics at the single organism level with cellular resolution are presented.

We decided to focus our attention on the nematode Caenorhabditis elegans. ${ }^{(4)}$ The many reasons to do so lean on those that originally convinced Brenner to select the worm as a basis for rational genomics research. ${ }^{(5)}$

- Standardisation. The embryonic development of C. elegans follows an identical map, such that cellular predecessors are exactly known. Responses are also largely programmable, such that a worm colony can be reasonably synchronised.

- Tractability. With only $\sim 10^{3}$ cells and 302 neurons, localisation is readily related to function as well as to behaviour.

- Transparency. Phenotypes are not always optically distinguishable, so that molecular phenotyping takes on a particular significance.

- Physical dimensions and other practicalities. The worm (see next section) has dimensions close to those of practical microfluidics and represents approximately the smallest organism size at which inductive NMR still has a scaling advantage. It has a very short lifecycle, allowing fast experimentation, and produces a large number of progenies. The worms can be maintained at $-80{ }^{\circ} \mathrm{C}$ and revived easily.

\section{NMR Signal Strength in the Context of Metabolomics}

The signal-to-noise ratio of the NMR experiment (see Sect. 4) is a fundamental quantity that describes the signal level that can be obtained from the sample, given the specific hardware choices, such as the size and arrangement of the detector, the polarisation field, and, of course, the sample type and concentration. Our purpose is to determine the signal intensity per unit sample volume in units that are easy to scale and assess. We consider the adult nematode C. elegans throughout, which has a length of about $1100 \mu \mathrm{m}$. The worm has a maximum 
diameter of about $80 \mu \mathrm{m}$, yielding a cross-sectional area of $\sim 5000 \mu \mathrm{m}^{2}$, and hence a volume of $\sim 5 \mathrm{pL}$ per $\mu \mathrm{m}$ of body length. The total body volume is therefore $\sim 5 \mathrm{~nL}$. Typical metabolite concentrations lie in the $\mathrm{mM}$ to $\mathrm{nM}$ range, from which we can determine the suitable signal intensities. For example, in C. elegans, a concentration of $1 \mathrm{nM}(\mu \mathrm{M}, \mathrm{mM})$ corresponds to $\sim 3.3 \times 10^{6}\left(10^{9}, 10^{12}\right)$ molecules or $\sim 10^{3}\left(10^{6}, 10^{9}\right)$ per cell. For an NMR limit of detection of $\sim 10^{12}$ molecules, this implies requiring $\sim 10^{6}\left(10^{3}, 10^{\circ}\right)$ worms for a distinguishable signal.

As we will see below, the best current NMR technology requires at least an entire worm to measure an NMR signal (Fig. 1), and this will yield data for only the most abundant metabolites, such as the major participants of central glycolysis and the Krebs cycle. This represents a reasonable starting point for in vivo metabolomics, since about $1 / 3$ of the worm body mass is muscle tissue. As we will also see below, it is possible to enhance the NMR signal, and the measures to improve the detectability (or reduce the lower limit of detection) can subsequently be used for one of the following possibilities:

- An extension in the number of detectable metabolites. Thus, more rare metabolites may become detectable, which in turn would reveal the dynamics of metabolomic cycles that involve these metabolites.

- A reduction in the compartment size. In this way, we may move from a detectable volume, the size of a worm, to a slice, and perhaps to a cell or an organelle. One would then be able to decipher the metabolomic rate at the level of this smaller compartment.

- A reduction in time resolution. If the detection of a well-resolved NMR signal together with signal recovery takes $\tau$ seconds, then $1 / \tau$ is the frequency at which we can determine the new concentration of a specific metabolite so that, following the Nyquist theorem, we can resolve the dynamics of the metabolite to, at most, a frequency of $1 /(2 \tau)$. For $\tau$, we have $\tau>2 \cdot \min \left(T_{1}\right.$, $\left.T_{2}\right)$.

\section{Types of NMR Experiments}

The magnetic resonance frequency $\omega=2 \pi \cdot f$ of an ensemble of nuclei with the gyromagnetic ratio $\gamma$ depends on the strength of the magnetic field $B_{0}$ they are subjected to through the

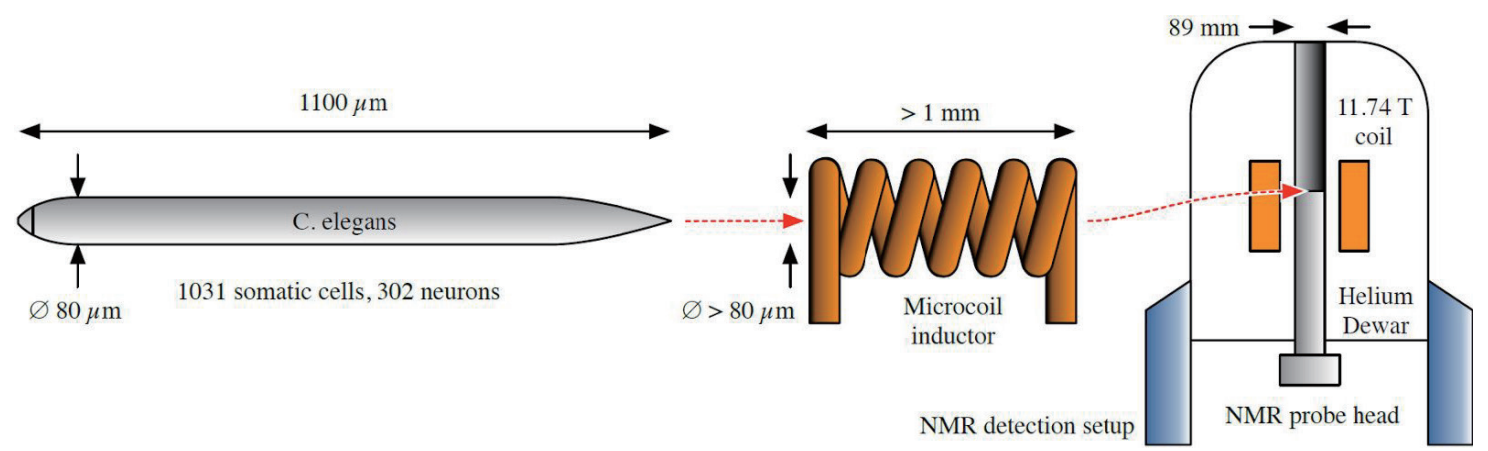

Fig. 1. (Color online) The C. elegans worm is placed within the windings of a microcoil resonator in the strong field of an NMR magnet for subsequent NMR spectroscopy or imaging. 
relation $\omega=\gamma \cdot B_{0}$. The frequency is primarily determined by the strength of the NMR magnet, which is built to have a highly uniform field distribution, so that any remaining differences in frequency only pertain to the sample, not the hardware. Sample-induced changes in magnetic field strength are caused by the local chemically defined magnetic environment in a particular molecule, referred to as chemical shift, a value that can be highly precisely determined for a particular chemical species. It is also possible to impose a coordinate-dependent magnetic field gradient that enables the spatial encoding of the signal. Further signals can be generated by correlating two or more frequencies mediated by internucleus couplings, and can be used to make very precise assignments of molecular structures and dynamics (see Spin Dynamics ${ }^{(6)}$ for details). Thus, magnetic resonance spectroscopy (MRS) primarily focuses on the frequencybased discrimination of nuclei and structural elucidation, and magnetic resonance imaging (MRI) primarily yields spatial distributions of spin density, although the two approaches are often combined. Solid samples require special treatment, because the lack of molecular tumbling causes resonance line broadening. The fast spinning of a solid sample at the magic angle with respect to $B_{0}$ has a similar effect of reducing the unwanted dipolar-dipolar coupling term, and poses a challenge for the spectroscopy of living samples.

\section{Signal-to-noise Ratio of Microdetectors}

The signal-to-noise (SNR) ratio of the inductive NMR experiment was first derived by Hoult and Richards, ${ }^{(7)}$ and is briefly repeated here for convenience. The measurable NMR signal is a time-dependent electromotive force generated across the terminals of the NMR detection coil and produced by an excited ensemble of nuclear spins whose net precessing magnetisation is

$$
M(t)=N \cdot \gamma^{2} \cdot \hbar^{2} \cdot I(I+1) B_{0} /\left(3 k_{B} \cdot T\right),
$$

where $N$ is the number of resonating spins per unit volume, $\gamma$ is the gyromagnetic ratio of the nuclei of interest, $\hbar$ is the reduced Planck's constant, $I$ is the spin quantum number, $B_{0}$ is the static magnetic field, and $T$ is the sample temperature. Besides these parameters, the strength of the NMR signal also depends on the volume of the detection coil, and on the coupling strength between the coil and the magnetisation, or, in other words, the filling factor of the coil. This dependence is governed by the reciprocity principle, ${ }^{(7)}$ which states that the time-dependent electromotive force generated across the terminals of the detection coil by the magnetisation $M(x, t)$ is directly proportional to the field per unit current at which that coil can produce for that specific magnetisation. From this, we can derive the relation for the signal for a coil with uniform $B_{1}$ :

$$
\xi(t)=\omega_{0} \cdot B_{1(x y)} \cdot M_{0} \cdot V_{s} \cdot \cos \omega_{0} t,
$$

where $\omega_{0}$ is the Larmor precessing frequency, $B_{1(x y)}$ is the transverse field per unit current of the detection coil, and $V_{s}$ is the sample volume. The coil of length $l$, resistivity $\rho$, permeability $\mu$, and wire perimeter $p$ has a finite radio-frequency resistance, $R_{c}=(l / p) \sqrt{\mu \cdot \mu_{0} \cdot \omega_{0} \cdot \rho\left(T_{c}\right) / 2}$, 
such that we can immediately postulate a Johnson/Nyquist noise voltage $\eta(t)$, which represents the amplitude of voltage fluctuation that is observable for an unbiased coil within a specific bandwidth $\Delta f$ :

$$
\eta(t)=\sqrt{4 k_{B} \cdot T_{c} \cdot \Delta f \cdot R_{c}} .
$$

The SNR therefore describes the extent to which a useful signal extends above the background noise signal, i.e., SNR $=\xi(t) / \eta(t)$. This formula sets the boundaries for the parameters that one can optimize in order to obtain a higher SNR.

\section{Signal Detection}

NMR signals of small samples can be detected in numerous ways, including variously arranged microcoil resonators, stripline resonators, superconducting quantum interference devices (SQUIDs), magnetic resonance force microscopy probes, and nitrogen vacancy centres in diamond. Here, we consider practical Faraday coil designs that are suitable for the worm geometry. Uniform fields can be achieved by the solenoid, Helmholtz, or saddle coil arrangements of the microwires. To achieve a greater SNR through miniaturisation, we note that the $B_{1}$-field scales inversely with the coil diameter $d$, i.e., $B_{1} \propto 1 / d$. Hence, we target uniform fields from coil windings placed close to the sample. The idea of signal improvement through miniaturisation has been around for quite a while. In 1979, Hoult and Lauterbur laid the foundation by theoretically deriving the scaling effects on the SNR. ${ }^{(8)}$ In 1995, Olson et al. used these scaling laws to build a hand-wound solenoidal coil with an inner diameter of 370 $\mu \mathrm{m}$ and thus obtained better or faster results on small (nl) samples. ${ }^{(9)}$ This study sets the upper sensitivity limit and in the many years since there were no feasible technological alternatives available that could beat this tiny hand-wound coil. In recent years, our group, as well as others, have developed a large variety of MEMS-derived techniques. These highly versatile and precise technologies have been modified to the needs of 3D coils, such as the automatic wirebonder, ${ }^{(10)}$ or a process called rolled-up-MEMS ${ }^{(11)}$ where a flexible substrate can be rolled up to yield a circular coil geometry. Examples of these are shown in Fig. 2, taken from previous publications. ${ }^{(11-15)}$ These technologies enable the fabrication of highly defined microcoils for a diameter range down to $\sim 300 \mu \mathrm{m}$. Moving beyond this limit is possible by using Lenz lenses, which act to focus the magnetic flux generated by the microcoil. These devices are capable of SNR improvements of approximately a factor of 3 on a sample volume that is $11 \%$ of the total sample volume of the Helmholtz microcoil presented in Fig. 2(b). ${ }^{(16,17)}$ With this toolbox at hand, the improvement of SNR in microcoils becomes again more of a numerical optimisation or design problem than a technological challenge.

\section{Signal Enhancement}

The following techniques, which we group here for convenience, can be used to enhance the NMR SNR ratio, thereby achieving a higher limit of detection. 


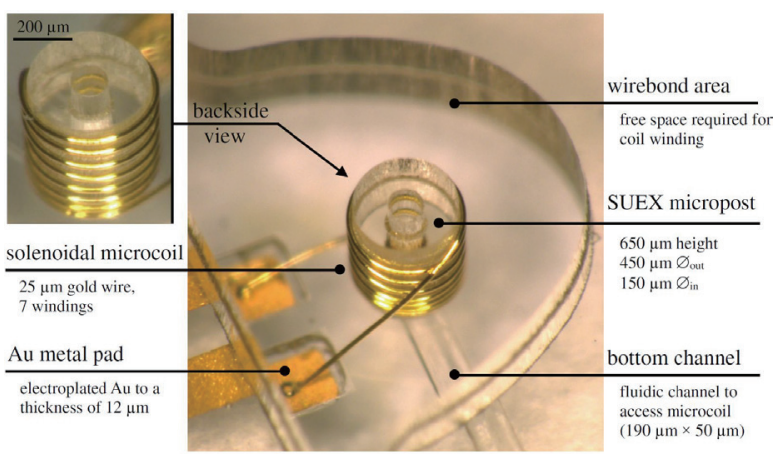

(a)
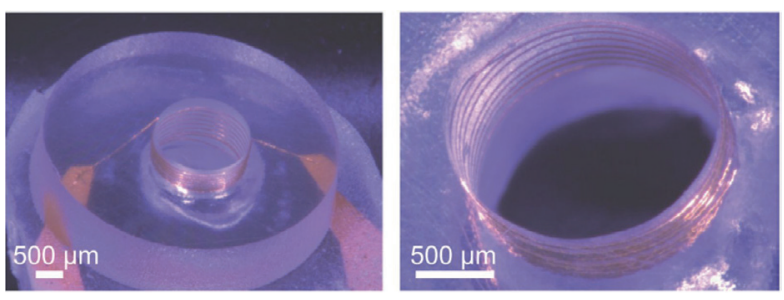

(c)

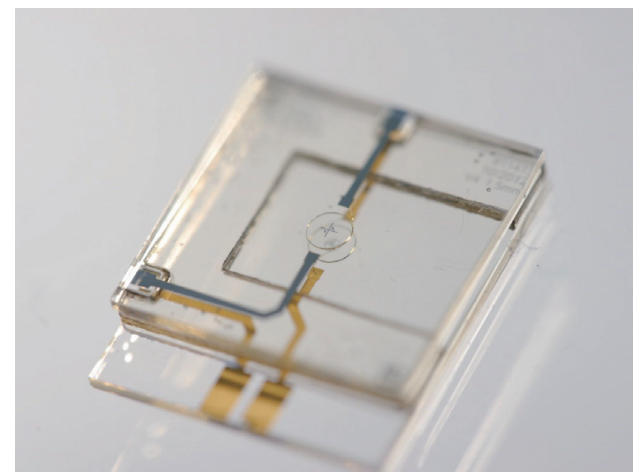

(b)

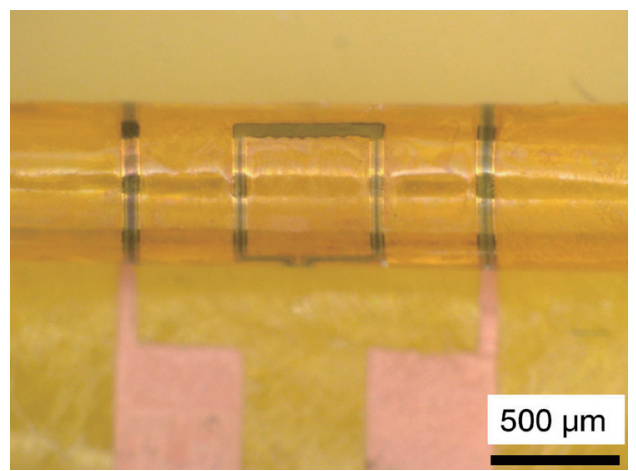

(d)

Fig. 2. (Color online) Four microcoil arrangements, with reported normalized limit of detections (nLOD) and spectral linewidths (LW) at a ${ }^{1} \mathrm{H}$ frequency of $500 \mathrm{MHz}$. (a) Solenoid (nLOD $4.86 \mathrm{nmol} \mathrm{Hz}^{-1 / 2}$, LW $8.0 \mathrm{~Hz}$ ) (C) IOP Publishing. Reproduced with permission. All rights reserved). ${ }^{(12)}$ (b) Helmholtz (nLOD $15.4 \mathrm{nmol} \mathrm{Hz}^{-1 / 2}$, LW 0.62 $\mathrm{Hz}) .^{(14,15)}$ (c) Hollow solenoid (LW $\left.40 \mathrm{~Hz}\right){ }^{(13)}$ (d) Saddle (nLOD $18.8 \mathrm{nmol} \mathrm{Hz}^{-1 / 2}$, LW $\left.1.5 \mathrm{~Hz}\right){ }^{(11)}$

- Use of stronger magnets. Normally, the NMR signal is induced by the strong field of a superconducting magnet. By doubling the field strength, for example, from $B_{0}=11.74 T$ to $\alpha_{B} \cdot B_{0}=23.58 T$, the NMR signal enhancement will become $\alpha_{B}{ }^{7 / 4}=3.36$.

- Cooling. At least for small-NMR samples, the noise is mainly produced by the detection hardware. By cooling the electronics to $\alpha_{T} \cdot T$, the SNR can be improved by a factor of $\alpha_{T}^{-1 / 2}$. For example, cooling from room temperature to liquid nitrogen temperature would enhance the SNR by 1.88. In NMR microscopy, it is, however, challenging to isolate a living sample from closely placed cold hardware.

- Oversampling. Multiple NMR signals are summed. For summed $n$ signals, the increase in SNR is proportional to $n^{1 / 2}$. However, the measurement then takes $n$ times longer, such that the time resolution of the experiment is sacrificed.

- Miniaturisation. As we have discussed, reducing the coil diameter by a factor of $\alpha_{d}$ results in an SNR improvement of $\alpha_{d}{ }^{-1}$. For example, compared with a $5 \mathrm{~mm}$ NMR detector, a microcoil of $0.5 \mathrm{~mm}$ diameter causes a tenfold enhancement of SNR. Radio-frequency phenomena such as the skin and proximity effects make it impractical to miniaturize detector coils with diameters below $200 \mu \mathrm{m}$ and wire thicknesses below $25 \mu \mathrm{m}$.

In practice, all of the the above enhancement factors can be combined, but even in combination, they yield only mild improvements in the C. elegans metabolomics experiment. More marked improvements are potentially possible through hyperpolarisation, and two techniques particularly show promise in the context of the worm. 
- Parahydrogen-induced polarisation in the form of signal amplification by reversible exchange $(S A B R E) .{ }^{(18)}$ In this modality, a source of ground-state para- $\mathrm{H}_{2}$ is needed and easily achievable at a sufficient quantity with a cryogenic setup. At room temperature, hydrogen has an equilibrium spin state distribution of $1 / 4$ para and 3/4 ortho, while at liquid helium temperature, the equilibrium is completely shifted in favour of the para state. The pure para- $\mathrm{H}_{2}$ represents a vast source of exploitable spin polarisation. Using a suitable catalyst, this spin order can be transferred from para- $\mathrm{H}_{2}$ to a desired substrate molecule. Hyperpolarised metabolites can then be tracked by NMR as they are metabolised by an organism, revealing their site and rate of consumption. ${ }^{(19)}$ Achievable signal enhancements with SABRE are in the 10-1000 range, depending on experimental details. Recent progress in the research on SABRE has also shown the potential for quantitative chemosensing in the micromolar and nanomolar metabolite concentration scales. ${ }^{(20)}$ In the context of miniaturised in situ and in vivo NMR, the main challenge in the implementation of para- $\mathrm{H}_{2}$ signal enhancement is in providing adequate amounts of para- $\mathrm{H}_{2}$ to sustain continuous hyperpolarisation throughout the timespan of the NMR experiment.

- Dynamic nuclear polarisation (DNP). ${ }^{(21)}$ By polarizing the electrons of radicals in a sample, dipole-dipole coupling and spin diffusion can be used to spread the polarisation outwards from the absorption site and across the sample's nuclei. At least two DNP variants are interesting and conducive to miniaturisation.

- In low-field Overhauser DNP, the sample is maintained at room temperature in the liquid state, whilst being subjected to microwave radiation, to achieve polarisation enhancements of ${ }^{13} \mathrm{C}$ of up to $1300 .{ }^{(22)}$ An interesting variant relies on the nitrogen vacancy centre in diamond, which has a spin-coupled optical transition, and hence can be optically pumped. This avoids the use of radicals, but requires nitrogen-vacancy (NV) centres within a few nanometres of the diamond surface, which is in contact with the sample. Polarisation enhancements of up to 2 orders of magnitude can be expected. ${ }^{(23)}$

- In high-field dissolution DNP, the sample is maintained at a very low temperature as a spin glass, subjected to microwave radiation, and then rapidly thawed to physiological temperatures before being introduced into the organism. ${ }^{(24)}$ Polarisation enhancements of up to 50,000 have been reported. ${ }^{(25)}$

\section{Signal Localisation Using Strong Gradients}

The chemical shift in the resonance of a particular nucleus, which is due to the molecular environment, can be modified to achieve spatial encoding. In essence, the resonance frequency of the nucleus $\omega=\gamma\left(B\left[x_{0}\right]+x \cdot \partial B / \partial x\right)$ is modified by a gradient term that is under experimental control. Gradient coils, arranged in three orthogonal directions, can be used to apply a strong magnetic field gradient that either selects a particular resonant slice or spatially encodes the NMR signal. In this way, the MRI of a particular atomic nucleus is facilitated, and the image contrast reflects one of various parameters, such as the spin density, or any of the various relaxation times. In metabolomics, slice selection is very useful in achieving a smaller compartment size and eliminating signals from background materials. Furthermore, gradients can be used in clever ways to achieve signal suppression of unwanted contributors, such as the water background, or other abundant but uninteresting molecules. 


\section{Mixture Analysis}

When performing NMR on C. elegans, all nuclei within the detector volume will normally contribute to the acquired signal, unless special precautions are taken. A naively recorded 1D NMR spectrum will therefore be an overlay of all the peaks from a very large number of molecules, including all lipids, proteins, DNA, and RNA. Additionally, the organism will most likely be in a nurturing environment, whose components will also contribute to the background signal. To assign the spectrum, that is, to identify the molecules contributing to the signal, the spectral overlap must be addressed. The spectral complexity is reduced simply because resonances emanating from molecules whose abundancies lie below the detection limit will appear as noise. For the remainder of the mixture contributing to the spectrum, special techniques are required to identify the components. In addition to the 1D NMR spectrum, multidimensional homonuclear (e.g., $\left.{ }^{1} \mathrm{H}-{ }^{1} \mathrm{H}\right)$ or heteronuclear $\left(\right.$ e.g., $\left.{ }^{1} \mathrm{H}-{ }^{13} \mathrm{C}\right)$ correlation spectra can be measured in order to reduce spectral overlay as signals are spread into multiple frequency dimensions. Preliminary molecular identification can then be done by comparison with metabolite spectral databases $\left(\mathrm{HMDB},{ }^{(26)} \mathrm{BMRB},{ }^{(27)} \mathrm{MMCD},{ }^{(28)}\right.$ and COLMAR ${ }^{(29)}$ ), followed by confirmation by authentic standard spiking. Further development in mixture analysis includes, as an example, the extraction of the spectral assignment from molecular spectral fingerprints derived from time-dependent coherence transfer within the molecule. ${ }^{(30)}$

\section{Conclusions}

In order to assess future solution strategies, the current limit of detection that is preventing the in vivo metabolomic monitoring of $C$. elegans requires qualification. For the single most abundant metabolite in the organism, we currently require the signal from at least one nematode. This means that less abundant metabolites or smaller compartments are not yet measurable with NMR. Only through massive signal oversampling at the expense of time resolution, this signal can be enhanced and hence localised into an interesting smaller compartment of the nematode, for example, in a slice or a single cell. We can therefore surmise that we are currently at the horizon of achieving a useful and hence biologically relevant result in terms of SNR and localisation. We have also learned that the inductively detected smallsample NMR experiment has reached its practical sensitivity limit, with very little hope for a marked improvement in SNR through further miniaturisation or redesign. ${ }^{(31)}$ Any improvement attempt must therefore either consider another more sensitive detection principle altogether, or the introduction of hyperpolarisation methods into biological samples. For example, a factor of $10^{3}$ in signal enhancement will render $\mathrm{mM}$ concentrations single-shot-detectable at the single cell level in $C$. elegans, but $\mu \mathrm{M}$ levels will still require raw oversampling factors of $10^{6}$, which is only feasible if sparse sampling is possible.

Our main conclusion, therefore, is that the NMR microscopy of C. elegans definitely holds promise for the fully resolved in vivo metabolomic profiling of an important model organism, but to do so will require at least additional three orders of magnitude in signal detectability, most likely achieved by hyperpolarisation. 


\section{Acknowledgments}

The work leading to this paper was primarily supported by the European Research Council (ERC) under grant number 290586 (NMCEL). Additional support was provided by the Deutsche Forschungsgemeinschaft (DFG), in the framework of the German Excellence Initiative under grant number EXC 1086 (BrainLinks-BrainTools), and grant number KO1883/23-1 (RUMS). The first author is greatly indebted to his hard-working team and collaborators who supported or contributed the numerous results that are summarized in this publication. Besides the co-authors, these include Shyam Adhikari, Natalia Bakhtina, Erwin Fuhrer, Andreas Greiner, Oliver Gruschke, Jürgen Hennig, Jens Höfflin, Jan Hövener, Robert Kamberger, Ronald Kampmann, David Kauzlaric, Sebastian Kiss, Mona Klein, Kai Kratt, Robert Meier, Markus Meissner, Ali Moazenzadeh, Nikolaus Nestle, Kirill Poletkin, Herbert Ryan, Pedro Silva, Christoph Trautwein, Marcel Utz, Ulrike Wallrabe, Nan Wang, Peter While, and Maxim Zaitzev.

\section{References}

1 M. Kanehisa and S. Goto: Nucleic Acids Res. 28 (2000) 27.

2 M. Kanehisa, S. Goto, Y. Sato, M. Kawashima, M. Furumichi, and M. Tababe: Nucleic Acids Res. 42 (2013) D199.

3 H. Kitano: Nature $\mathbf{4 2 0}$ (2002) 206.

4 WormBook: The C. elegans Research Community, WormBook, http://www.wormbook.org (accessed June 2017).

5 S. Brenner: Genetics 77 (1974) 71.

6 M. H. Levitt: Spin Dynamics: Basics of Nuclear Magnetic Resonance (John Wiley \& Sons, 2008).

7 D. I. Hoult and R. E. Richards: J. Magn. Reson. 24 (1976) 71.

8 D. I. Hoult and P. C. Lauterbur: J. Magn. Reson. 34 (1979) 425.

9 D. L. Olson, T. L. Peck, A. G. Webb, R. L. Magin, and J. V. Sweedler: Science 270 (1995) 1967.

10 K. Kratt, V. Badilita, J. G. Korvink, and U. Wallrabe: J. Micromech. Microeng. 20 (2010) 015021.

11 N. Wang, M. V. Meissner, N. MacKinnon, D. Mager, and J. G. Korvink: J. Micromech. Microeng. (in press).

12 R. Ch. Meier, J. Höfflin, V. Badilita, U. Wallrabe, and J. G. Korvink: J. Micromech. Microeng. 24 (2014) 045021.

13 R. Kamberger, A. Moazenzadeh, J. G. Korvink, and O. Gruschke: J. Micromech. Microeng. 26 (2016) 065002.

14 N. Spengler, A. Moazenzadeh, R. Ch. Meier, V. Badilita, J. G. Korvink, and U. Wallrabe: J. Micromech. Microeng. 24 (2014) 034004.

15 N. Spengler, J. Höfflin, A. Moazenzadeh, D. Mager, N. MacKinnon, V. Badilita, U. Wallrabe, and J. G. Korvink: PLoS ONE 11 (2016) e0146384.

16 N. Spengler, P. T. While, M. V. Meissner, U. Wallrabe, and J. G. Korvink: PLoS ONE 12 (2017) e0182779.

17 M. Jouda, R. Kamberger, J. Leupold, N. Spengler, J. Hennig, O. Gruschke, and J. G. Korvink: Concept Magnetic Res. 1 (2017) e21357.

18 R. W. Adams, J. A. Aguilar, K. D. Atkinson, M. J. Cowley, P. I. P. Elliott, S. B. Duckett, G. G. R. Green, I. G. Khazal, J. López-Serrano, and D. C. Williamson: Science 323 (2009) 1708.

19 N. M. Zacharias, H. R. Chan, N. Sailasuta, B. D. Ross, and P. Bhattacharya: J. Am. Chem. Soc. 134 (2012) 934.

20 N. K. J. Hermkens, N. Eshuis, B. J. A. van Weerdenburg, M. C. Feiters, F. P. J. T. Rutjes, S. S. Wijmenga, and M. Tessari: Anal. Chem. 88 (2016) 3406.

21 A. Abragam and M. Goldman: Rep. Prog. Phys. 41 (1978) 395.

22 M. D. Lingwood and S. Han: J. Magn. Reson. 201 (2009) 137.

23 C. O. Bretschneider, Ü. Akbey, F. Aussenac, G. L. Olsen, A. Feintuch, H. Oschkinat, and L. Frydman: Chem. Phys. Chem. 17 (2016) 2691.

24 R. E. Hurd, Y.-F. Yen, A. Chen, and J. H. Ardenkjaer-Larsen: J. Magn. Reson. Imag. 36 (2012) 1314. 
25 L. F. Pinto, I. Marin-Montesinos, V. Lloveras, J. L. Munos-Gómez, M. Pons, J. Veciana, and J. Vidal-Gancedo: Chem. Commun. 53 (2017) 3757.

26 D. S. Wishart, T. Jewison, A. C. Guo, M. Wilson, C. Knox, Y. Liu, Y. Djoumbou, R. Mandal, F. Aziat, E. Dong, S. Bouatra, I. Sinelnikov, D. Arndt, J. Xia, P. Liu, F. Yallou, T. Bjorndahl, R. Perez-Pineiro, R. Eisner, F. Allen, V. Neveu, R. Greiner, and A. Scalbert: Nucl. Acids Res. 41 (2013) D801.

27 E. L. Ulrich, H. Akutsu, J. F. Doreleijers, Y. Harano, Y. E. Ioannidis, J. Lin, M. Livny, S. Mading, D. Maziuk, Z. Miller, E. Nakatani, C. F. Schulte, D. E. Tolmie, R. K. Wenger, H. Yao, and J. L. Markley: Nucl. Acids Res. 36 (2007) D402.

28 Q. Cui, I. A. Lewis, A. D. Hegeman, M. E. Anderson, J. Li, C. F. Schulte, W. M. Westler, H. R. Eghbalnia, M. R. Sussman, and J. L. Markley: Nat. Biotechnol. 26 (2008) 162.

29 F. Zhang, S. L. Robinette, L. Bruschweiler-Li, and R. Brüschweiler: Magn. Reson. Chem. 47 (2009) S118.

30 N. MacKinnon, P. T. While, and J. G. Korvink: J. Magn. Reson. 217 (2016) 147.

31 V. Badilita, R. Ch. Meier, N. Spengler, U. Wallrabe. M. Utz, and J. G. Korvink: Soft Matter 8 (2012) 10583. 